\title{
Neural Stem Cell Therapy for Temporal Lobe Epilepsy
}

\author{
Ashok K. Shetty \\ Division of Neurosurgery, Duke University \& Veterans Affairs Medical Centers, Durham, NC \\ 27710, USA
}

\section{Summary}

Neural stem cells (NSCs) are one of the candidates for grafting in the realm of cell-based therapy for temporal lobe epilepsy (TLE). Because NSCs can produce neurons synthesizing the inhibitory neurotransmitter GABA and cells secreting multiple neurotrophic factors, NSC grafting therapy has promise for restraining seizures and easing cognitive dysfunction in TLE. For an expanded treatment of this topic see Jasper's Basic Mechanisms of the Epilepsies, Fourth Edition (Noebels JL, Avoli M, Rogawski MA, Olsen RW, Delgado-Escueta AV, eds) published by Oxford University Press. Available on NCBI Bookshelf.

\section{Keywords}

transplantation; GABA; glial-derived neurotrophic factor; hippocampus; seizures

Cell transplantation strategy has received significant attention as an alternative therapy for temporal lobe epilepsy (TLE) in pre-clinical studies. Neural stem cells (NSCs) are attractive as donor cells for grafting in TLE because these cells can be expanded in culture for extended periods from diverse sources such as the fetal, postnatal and adult brain, human embryonic stem cells, and human induced pluripotent stem cells. Moreover, NSCs can generate both neurons synthesizing the inhibitory neurotransmitter GABA and glia secreting multiple neurotrophic factors including glial-derived neurotrophic factor (GDNF), which has anticonvulsant properties.

Several studies have investigated the efficacy of NSC grafts for restraining seizures in different models of TLE through quantification of the behavioral spontaneous seizures. Some studies have examined the effects of grafting of NSCs derived from the fetal hippocampus or the adult anterior subventricular zone into hippocampi shortly after injury, status epilepticus or kindling. The results were promising, as grafting decreased the occurrence and/or intensity of spontaneous seizures (Jing et al., 2009. Kuruba et al., 2009, Shindo et al., 2010). Another study demonstrated that grafting of NSCs derived from the embryonic medial ganglionic eminance into hippocampi of rats that had chronic epilepsy for prolonged periods led to considerable reductions in both frequency and intensity of spontaneous seizures (Waldau et al., 2008). The NSC grafts also added significant numbers of new GABA+ neurons and GDNF+ astrocytes into the epileptic hippocampus, and normalized GDNF expression in the host astrocytes. From the perspective of the inhibitory function of GABA and the anticonvulsant property of GDNF, it was suggested that additions of new GABAergic neurons and GDNF+ astrocytes, and restoration of GDNF in the host astrocytes underlie seizure suppression mediated by NSC grafts.

Corresponding author: Ashok K. Shetty, M.Sc., Ph.D. Ashok.Shetty@ Duke.Edu.

Disclosure: The author declares no conflicts of interest. 
Thus, NSC grafting strategy has considerable promise for treating TLE. However, additional studies are needed prior to its clinical application. Grafting studies in chronic epilepsy prototypes using different types of NSCs, long-term video-EEG recordings, and rigorous graft-host integration analyses are critical to ascertain the extent, durability and mechanisms of seizure suppression mediated by NSC grafts. The ability of NSC grafts for reversing the cognitive dysfunction in TLE will also need to be assessed.

\section{Acknowledgments}

Supported by a grant from the NIH-NINDS (RO1 NS054780 to A.K.S).

\section{References}

Jing M, Shingo T, Yasuhara T, Kondo A, Morimoto T, Wang F, Baba T, Yuan WJ, Tajiri N, Uozumi T, Murakami M, Tanabe M, Miyoshi Y, Zhao S, Date I. The combined therapy of intrahippocampal transplantation of adult neural stem cells and intraventricular erythropoietin-infusion ameliorates spontaneous recurrent seizures by suppression of abnormal mossy fiber sprouting. Brain Res. 2009; 1295:203-217. [PubMed: 19646969]

Kuruba R, Hattiangady B, Shuai B, Shetty AK. Effects of grafting of hippocampal stem/progenitor cells shortly after status epilepticus on the development of chronic epilepsy. Cell Transplant. 2009; 18:221-221.

Shindo A, Nakamura T, Matsumoto Y, Kawai N, Okano H, Nagao S, Itano T, Tamiya T. Seizure suppression in amygdala-kindled mice by transplantation of neural stem/progenitor cells derived from mouse embryonic stem cells. Neurol Med Chir. 2010; 50:98-105.

Waldau B, Hattiangady B, Kuruba R, Shetty AK. Medial ganglionic eminence-derived neural stem cell grafts ease spontaneous seizures and restore GDNF expression in a rat model of chronic temporal lobe epilepsy. Stem Cells. 2010; 28:1153-1164. [PubMed: 20506409] 